\title{
Understanding biology of potato- virus PUY interaction
}

\section{Kristina Gruden}

National Institute of Biology, Ljubljana, Slovenia

\section{Motivation and Objectives}

In nature plants encounter various factors, which influence their growth and development and consequently affect plant product quantity and quality. Potato virus $Y(P V Y)$ is a severe plant pathogen responsible for yearly losses in production of Solanaceae crops worldwide. Plant responses to viruses and the disease development are different and much less explored in comparison to bacterial or fungal infections. In single component studies the complexity of the plant - pathogen interaction at molecular level can lead to limited conclusions that may fail to notice important changes in physiological processes. Omics approaches, that offer a more holistic view of the processes, are therefore a major step forward in understand these interactions. In addition, we are aiming at identifying novel components of plant defence like noncoding RNAs, potato endo- and epiphytes as well as estimate sequence variability on the pathogen site.

\section{Methods}

In our studies, gene expression in the disease response of the susceptible, tolerant and resistant potato (Solanum tuberosum L.) cultivars to PVY infection was investigated at different times after infection, using transcriptomics approaches.

Most of our studies were performed combining microarrays and real-time PCR for transcriptomics. Recently, we have complemented our results with the RNA-sea analysis on Solid platform. In addition, we have performed analysis of small RNAs libraries both on Solid as well as on Illumina platform.

\section{Results and Discussion}

With 'standard' transcriptomic approaches we have shown that not only the components involved but also the timing and intensity of response are extremely important for the outcome of plant-virus interaction. Small RNA analysis using Solid platform identified significant differences of several miRNAs after viral infection that were so far not implied in plant defence against viruses. Results of other NGS related experiments are still in progress. 